\title{
Synthesis and characterization of tungstophosphoric acid-modified mesoporous silica nanoparticles with tuneable diameter and pore size distribution
}

\author{
Alexis A. Sosa ${ }^{1} \cdot$ Marina N. Gorsd ${ }^{1} \cdot$ Mirta N. Blanco $^{1} \cdot$ Luis R. Pizzio $^{1}$
}

Received: 7 April 2017 / Accepted: 16 May 2017 / Published online: 25 May 2017

(C) Springer Science+Business Media New York 2017

\begin{abstract}
Mesoporous silica nanoparticles were prepared in aqueous/organic phase using cetyltrimethylammonium bromide and polystyrene as organic templates. The morphology and crystalline phase of the products were characterized by scanning electron microcopy, transmission electron microscopy, X-ray diffraction, small angle X-ray scattering, and $\mathrm{N}_{2}$ adsorption/desorption isotherm analysis. The octane/water ratio influenced the pore size distribution, the morphology and size of the nanospheres obtained. Transmission electron microscopy revealed that mesoporous silica nanoparticles with "blackberry-like structure" (MSN3, MSN4, MSN5, and MSN6 samples) were obtained using octane/water ratios in the range 0.007-0.35. They present small (in the range 5-6 $\mathrm{nm}$ ) and large (in the range 28-34 nm) mesopores. Large mesopores were mainly generated by polystyrene, and their volume contribution was clearly higher than in the MSN1 and MSN2 samples. The structure and morphology of mesoporous silica nanoparticles solids impregnated with tungstophosphoric acid were similar to those of the mesoporous silica nanospheres used as support. In addition, the characterization of all the solids impregnated with tungstophosphoric acid by Fourier transform infrared and ${ }^{31} \mathrm{P}$ nuclear magnetic resonance indicated the presence of undegraded $\left[\mathrm{PW}_{12} \mathrm{O}_{40}\right]^{3-}$ and
\end{abstract}

Electronic supplementary material The online version of this article (doi:10.1007/s10971-017-4428-6) contains supplementary material, which is available to authorized users.

Luis R. Pizzio

1rpizzio@quimica.unlp.edu.ar

1 Centro de Investigación y Desarrollo en Ciencias Aplicadas "Dr. Jorge J. Ronco" (CINDECA), Departamento de Química, Facultad de Ciencias Exactas, Universidad Nacional de La Plata-CCT La Plata, CONICET, Calle $47 N^{\circ}$ 257, La Plata 1900, Argentina
$\left[\mathrm{H}_{3-X} \mathrm{PW}_{12} \mathrm{O}_{40}\right]^{(3-X)-}$ species interacting electrostatically with the $\equiv \mathrm{Si}-\mathrm{OH}_{2}{ }^{+}$groups, and by potentiometric titration the solids presented very strong acid sites. In summary, they are good candidates to be used in reactions catalyzed by acids, especially to obtain quinoxaline derivatives.

\section{Graphical Abstract}

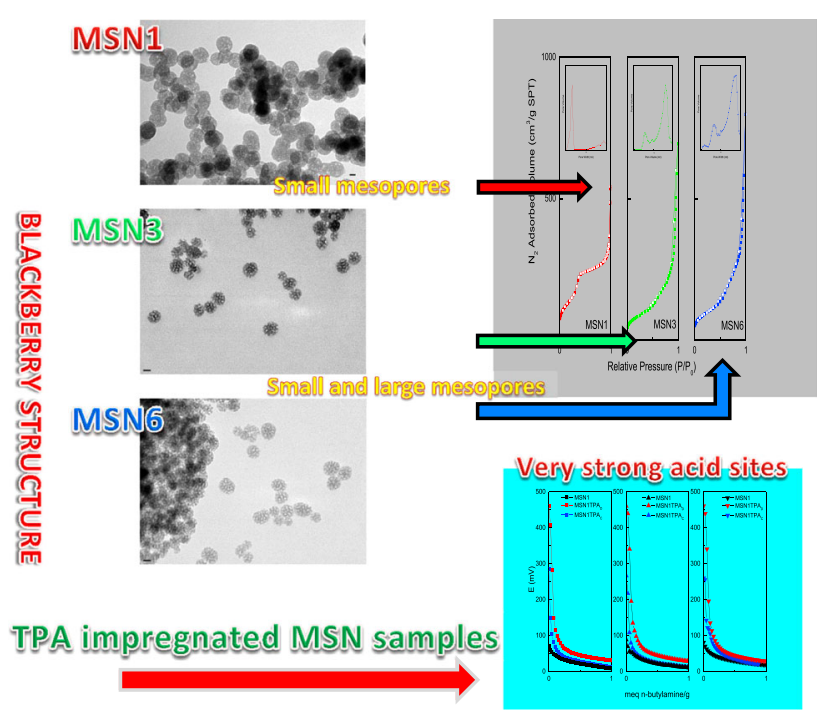

Keywords Nanoparticles · Silica - Mesoporous solids • Tungstophosphoric acid

\section{Introduction}

Recently, much has been reported about the synthesis and application of mesoporous materials in different science areas $[1,2]$. As these materials have a wide use in diverse 
fields, increasing efforts have been made to find new and better preparation routes $[3,4]$.

Within the spectrum of materials with high importance in nanotechnology, one of the most outstanding is mesoporous silica, which has attracted much interest in a few years as it is widely used because it is cheap, safe, chemically inert, thermally stable, and biocompatible [5]. Due to the quickness of technological advances, there are a number of techniques available for the preparation of nanostructured materials, which can be divided into three categories, according to the preparation route. Among them, the liquid preparation method has some advantages over the others, such as low cost, increased productivity, and environmental friendliness, among others.

Reports collected from the literature have explored the influence of the size, shape, functionalization and use of these structurally ordered materials for application in a variety of fields, such as catalysis [6], drug transport [7], separations [8], electronic optics [3], and also as sensors or adsorbents [9]. The most widely known and studied mesoporous silica nanoparticles (MSN) are those named MCM-41 and SBA-15, which possess a mesoporous ordered structure and a uniform pore size [10]. Besides, techniques for the preparation of mesoporous siliceous materials with different morphologies, such as spheres, films, fibers, among others, are currently being explored [11]. Many of these methods have proved to be useful for industrial applications. However, some disadvantages still remain, such as the use of harmful chemical products, which can pose a problem for bioapplications, and something very important in heterogeneous catalysis such as the pore size, which may create difficulties for the reactant penetration into the catalytic material and the diffusion of reactants or products to or from the active sites [12]. It is known that the morphological characteristics such as size and shape can be adjusted by kinetic control of the reaction; this is the reason for using templates such as cationic or nonionic surfactants, polymers or electrolytes, among others. Many researchers have reported the preparation of particles with controllable morphology through liquid phase synthesis [12] and also by the spray method [3].

Among the possible morphologies, the spherical structure of the MSN has attracted much interest and has been prepared through various methods, such as sol-gel [13], microemulsion [14], template addition [15], to mention the most widely used. An important challenge is to obtain silica nanospheres with controllable particle and pore size by the template method in liquid phase. Nandiyanto et al. [12] described the synthesis of mesoporous silica with adjustable pore size (from 4 to $15 \mathrm{~nm}$ ) and outer particle diameter (from 20 to $80 \mathrm{~nm}$ ). The employed method involved a reaction system of double synthesis within the micelle; one is the hydrolysis reaction of tetraethylorthosilicate (TEOS) to produce the silica and the other, styrene polymerization, subsequently followed by the removal of the organic templates (cetyltrimethylammonium bromide (CTAB) and polystyrene (PS)) through calcination.

On the other hand, the heteropolyacids (HPA) with wellknown Keggin structure [16] can be used in their bulk form both in homogeneous and heterogeneous catalysis, though it is advisable to support the HPA because the bulk compounds present a very low specific surface area $\left(3-5 \mathrm{~m}^{2} / \mathrm{g}\right)$, which limits their use in this manner. To overcome this disadvantage, the HPA are dispersed on a support such as silica, zirconia, alumina or carbon, among others. According to the preparation method, the HPA interact with the surface when they are supported on zirconia, alumina or carbon, and the Keggin structure can be partially decomposed, resulting in relatively low acidic materials [17, 18]. On the other hand, it was reported that the HPA are more stable when supported on silica $[19,20]$. It is known that there is an electrostatic interaction between the heteropolyacid and the support, which occurs through the protonation of the surface hydroxyl groups [21]. Legagneux et al. [22] supported different kinds of HPA on silica, and proposed the mode of interaction with silanol groups. The characteristics of the HPA/support solids obtained by impregnation techniques depend on the nature and stability of the species during impregnation, drying and calcination steps [23], and thus the catalytic properties of the catalysts also depend on these processes. During the impregnation, the contact between the support and the solution, the adsorption of the active species precursors is one of the most important factors to be considered.

The use of supported HPA and related compounds in heterogeneous catalysis is known and of great importance, becoming increasingly attractive. These solids have advantages compared with the homogeneous catalysts, such as the easy separation from the reaction medium and the possibility of reuse in liquid phase reactions, without a great change in the reaction yield [4]. Thus, they are a very good substitute to be used in acidic reactions instead of the more common mineral acids (sulfuric acid, hydrochloric acid, nitric acid, among others), which involve the generation of massive amounts of toxic wastes at an industrial scale. Several authors have explored the application of supported HPA in diverse catalyzed reactions [24-27]. It is a desirable topic to consider in order to promote ecocompatible synthetic routes as proposed by the so-called Green Chemistry [28].

The objective of this work is to prepare and characterize mesoporous silica nanospheres obtained in aqueous/organic phase by the template method, with the aim of achieving a support with appropriate morphology and textural properties. Then, the nanospheres are impregnated with tungstophosphoric acid (TPA) in order to obtain materials with 
suitable textural and acidic characteristics for further use in heterogeneously catalyzed reactions for the synthesis of quinoxaline derivatives with biological applicability.

\section{Experimental}

\subsection{Support preparation}

The mesoporous silica nanoparticles (MSNX) were prepared by TEOS hydrolysis, adding lysine as catalyst, cetyltrimethyilammonium bromide (CTAB) as template and surfactant, styrene as the template precursor, and cyanovaleric acid (ACVA) as initiator of styrene polymerization. In a typical process to prepare the silica/template particles, $0.35 \mathrm{~g}$ of CTAB was first dissolved in $108 \mathrm{ml}$ of water at $60{ }^{\circ} \mathrm{C}$ in a three-necked flask. When a clear solution was obtained, octane $(32 \mathrm{ml})$, styrene $(42 \mathrm{mg})$, lysine $(80 \mathrm{mg})$, TEOS ( $3.5 \mathrm{~g})$, and ACVA $(90.5 \mathrm{mg}$ ) were added. The reaction was continued for $3 \mathrm{~h}$ under $\mathrm{N}_{2}$ atmosphere at $60{ }^{\circ} \mathrm{C}$. Then, the heating was stopped, and the suspension was cooled at room temperature, allowed to stand for $18 \mathrm{~h}$, and later, the solid was isolated by centrifugation. This material was washed with ethanol and finally, the template was removed by thermal treatment at $500{ }^{\circ} \mathrm{C}$ for 3 h. Samples were prepared by varying the styrene/water $\left(\mathrm{EST} / \mathrm{H}_{2} \mathrm{O}\right)$ mass ratio in the range 0.39-10, keeping the ACVA amount constant, or by varying the ratio of the organic/aqueous phases, employing octane/water $\left(\mathrm{OCT} / \mathrm{H}_{2} \mathrm{O}\right)$ mass ratios between 0.0006 and 0.35 , with the purpose of observing the effect of these variables on the characteristics of the obtained materials.

\subsection{Impregnation with TPA}

The experiments were performed by contacting, at room temperature, $0.7 \mathrm{~g}$ of the support (MSNX) with $0.3 \mathrm{~g}$ of $\operatorname{TPA}\left(\mathrm{H}_{3} \mathrm{PW}_{12} \mathrm{O}_{40}\right)$ dissolved in $3 \mathrm{ml}$ of ethanol-water $50 \%$ (v/v) solution in order to obtain a TPA concentration of $30 \%$ by weight in the final material. The solution and the support were allowed to stand in contact until the solvent was evaporated and the solid was dried at $70^{\circ} \mathrm{C}$. Finally, the solid was calcined at $200^{\circ} \mathrm{C}$ for $2 \mathrm{~h}$ under air atmosphere, thus obtaining the solids that will be named MSNXTPA . $_{\text {. }}$ The TPA content in the samples was estimated as the difference between the $\mathrm{W}$ amount contained in the TPA solution originally used for the impregnation and the amount of $\mathrm{W}$ that remained in the beaker after removing the dried samples. Additionally, in order to remove the TPA weakly bound to the MSNX surface, the materials were contacted with water (typically $0.100 \mathrm{~g}$ in $10 \mathrm{ml}$ ) for $24 \mathrm{~h}$, filtered, and dried at $100{ }^{\circ} \mathrm{C}$. The amount of $\mathrm{W}$ in the obtained solutions was determined by atomic absorption spectrometry using a Varian AA Model 240 spectrophotometer. The calibration curve method was used with standards prepared in the laboratory. The analyses were carried out at a wavelength of $254.9 \mathrm{~nm}$, bandwidth $0.3 \mathrm{~nm}$, lamp current $15 \mathrm{~mA}$, phototube amplification $800 \mathrm{~V}$, burner height $4 \mathrm{~mm}$, and acetylene-nitrous oxide flame (11:14).

\subsection{Characterization}

The nitrogen adsorption/desorption measurements were carried out at liquid nitrogen temperature $(77 \mathrm{~K})$ using Micromeritics ASAP 2020 equipment. From the obtained data, the specific surface area $\left(S_{\mathrm{BET}}\right)$, using the Brunauer-Emmett-Teller model in the relative pressure range $0.05-0.3$, the mean pore diameter $(\mathrm{Dp})$ by the $\mathrm{BJH}$ method, and the pore size distribution (PSD) with the DFT density functional theory (DFT) method were estimated.

The morphology and the size of the MSNX were characterized by scanning electron microscopy (SEM) with Philips 505 equipment, and transmission electron microscopy (TEM) was performed on JEOL 100 CX II equipment, with an acceleration voltage of $100 \mathrm{kV}$ and a magnification range of $270,000 \times-450,000 \times$. The energy dispersive X-ray analysis (EDX) of the samples was obtained using an EDAX 9100 analyzer at a working potential of $15 \mathrm{kV}$.

The species present in the prepared samples were evaluated by Fourier transform infrared spectroscopy (FT-IR), using Bruker IFS 66 equipment with pellets of the sample in $\mathrm{KBr}$, in the $400-4000 \mathrm{~cm}^{-1}$ range at room temperature.

The solids impregnated with TPA were analyzed by ${ }^{31} \mathrm{P}$ magic angle spinning-nuclear magnetic resonance (MAS-NMR) spectroscopy before and after being calcined at $200{ }^{\circ} \mathrm{C}$. For this purpose, Bruker MSL-300 equipment was employed, using $5 \mu$ s pulses, a repetition time of $3 \mathrm{~s}$, and working at a frequency of $121.496 \mathrm{MHz}$ for ${ }^{31} \mathrm{P}$ at room temperature, the resolution being $3.052 \mathrm{~Hz}$ per point. A 5 $\mathrm{mm}$ diameter and $10 \mathrm{~mm}$ high sample holder was used, the spin rate was $2.1 \mathrm{kHz}$. Several hundred pulse responses were collected. Phosphoric acid $85 \%$ was employed as external reference.

The structural characteristics were determined by X-ray diffraction (XRD) employing Philips PW-1732 equipment, using $\mathrm{Cu} \mathrm{K} \alpha$ radiation, Ni filter, $20 \mathrm{~mA}$ and $40 \mathrm{kV}$ in the high voltage source, a scanning range between 5 and $60^{\circ}$ $2 \theta$, and a scanning rate of $2 \% \mathrm{~min}$. The small angle XRD patterns were recorded using Philips APD 1700 XPERT equipment with a built-in recorder. The conditions used were: $\mathrm{Cu} \mathrm{K} \alpha$ radiation, $\mathrm{Ni}$ filter, $40 \mathrm{~mA}$ and $40 \mathrm{kV}$ in the high voltage source, scanning range from 0.3 to $5^{\circ} 2 \theta$, and a scanning speed of $0.01 \%$.

The small angle X-ray scattering (SAXS) analysis was carried out to study the structural properties of MSNX 


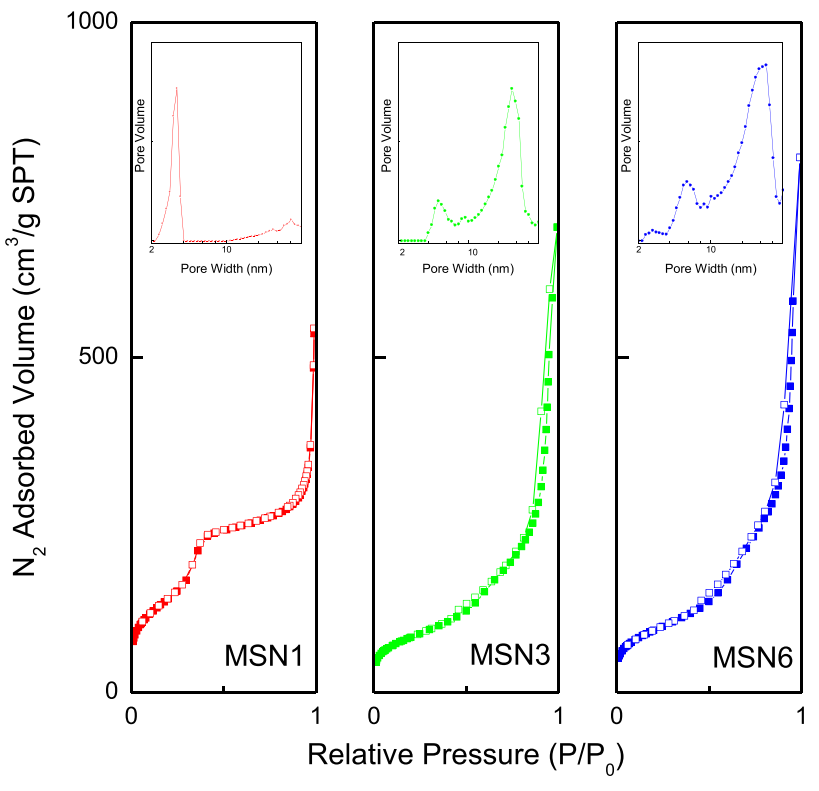

Fig. $1 \mathrm{~N}_{2}$ adsorption-desorption isotherms and pore size distribution (insert) of MSN1, MSN3, and MSN6 samples

samples. The dispersion curves of intensity $I$ (q) were determined as a function of the scattering vector (q) module. The SAXS measurements were performed using a laboratory SAXS setup (XEUSS 1.0, XENOCS, Grenoble) at $21{ }^{\circ} \mathrm{C}$ in the transmission configuration with variable sample-detector distance (in order to cover the angular range required for analysis).

The acidic properties of the solids were estimated by potentiometric titration, suspending the solid in acetonitrile and titrating with an $n$-butylamine solution in acetonitrile. The potential variation was measured employing a Hanna $211 \mathrm{pH}$ meter, with a double junction electrode.

\section{Results and discussion}

The $\mathrm{N}_{2}$ adsorption-desorption isotherms of representative MSNX silica samples are shown in Fig. 1, and the most important textural data are listed in Table 1. The isotherms of MSN1 and MSN2 samples can be classified as type IV, characteristic of mesoporous materials. They present a comparatively sharp inflection in the $0.30-0.42$ range of $P / P_{\mathrm{o}}$ relative pressure without hysteresis, assigned to the capillary condensation of $\mathrm{N}_{2}$ in ordered mesoporous materials [29]. The increase of $\mathrm{N}_{2}$ adsorbed in the 0.8-1 range of $P / P_{\mathrm{O}}$ is associated with the presence of large mesopores. The PSD obtained by the DFT method (graph inserted in Fig. 1) shows that the pores are mainly between 2 and $4 \mathrm{~nm}$ in size (centered at $\sim 3.4 \mathrm{~nm}$ ). Large mesopores (between 12 and $50 \mathrm{~nm}$ ) are also present, although their pore volume is rather small.
Table 1 Textural properties and size of representative MSN

\begin{tabular}{llllll}
\hline Sample & $\begin{array}{l}\mathrm{EST} / \mathrm{H}_{2} \mathrm{O} \\
\text { ratio }(w / w)\end{array}$ & $\begin{array}{l}\mathrm{OCT} / \mathrm{H}_{2} \mathrm{O} \\
\text { ratio }(\mathrm{w} / \mathrm{w})\end{array}$ & $\begin{array}{l}S_{\mathrm{BET}} \\
\left(\mathrm{m}^{2} / \mathrm{g}\right)\end{array}$ & Dp (nm) & $\begin{array}{l}\text { Size } \\
(\mathrm{nm})\end{array}$ \\
\hline MSN1 & 0.39 & 0.0006 & 497 & 5.9 & 55.7 \\
MSN2 & 0.39 & 0.0008 & 513 & 5.7 & 55.0 \\
MSN3 & 0.39 & 0.07 & 293 & 13.5 & 30.7 \\
MSN4 & 0.39 & 0.21 & 329 & 13.3 & 31.8 \\
MSN5 & 0.39 & 0.35 & 421 & 13.9 & 32.2 \\
MSN6 & 10 & 0.35 & 557 & 12.0 & 28.1 \\
\hline
\end{tabular}

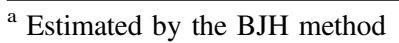

The isotherms of MSN3, MSN4, MSN5, and MSN6 samples (Fig. 1) can also be classified as type IV; however, they do not display the sharp inflection in the $0.30-0.42$ range of $P / P_{\mathrm{o}}$ relative pressure, and the capillary condensation step in the $0.8-1$ range of $P / P_{\mathrm{o}}$ is clearly higher (with $\mathrm{H} 1$ hysteresis loops). The PSD also reveals the presence of small (centered at $\sim 5.8 \mathrm{~nm}$ ) and large mesopores (centered in the range $28-34 \mathrm{~nm}$ ) in these samples. For these materials, the volume of large mesopores is clearly higher than in the MSN1 and MSN2 samples. As can be observed (Table 1), all the samples present high specific surface area. Additionally, the specific micropore area values, estimated from the $t$-plot method, show that more than $95 \%$ of the total surface area comes from a mesoporous structure.

The SEM micrographs of MSN1, MSN2, and MSN3 samples (Fig. 2) show that the materials are formed by agglomerates of spherical particles. The size of these spherical particles (in the range 200-100 nm) decreases slowly in the following order MSN1 $>$ MSN2 $>$ MSN3. The SEM micrographs of the other three samples reveal that their size and morphology are similar to those of MSN3 material.

The spherical particles seem to be formed by aggregation of smaller nanoparticles that, according to the TEM micrographs (Fig. 3), also have spherical shape with a size between 55 and $28 \mathrm{~nm}$. In the case of the MSN3, MSN4, MSN5, and MSN6 samples, TEM micrographs show that they are formed by clusters of $5 \mathrm{~nm}$ nanoparticles, giving them the appearance of a blackberry.

The increment of the OCT/ $\mathrm{H}_{2} \mathrm{O}$ ratio from 0.0008 to 0.07 led to a significant change of the PSD, morphology and size of the nanospheres obtained. However, they remained almost constant when the ratio was raised to 0.21 or 0.35 . Mesoporous silica nanospheres (size $\sim 55 \mathrm{~nm}$ ) with Dp in the range $5.7-5.9 \mathrm{~nm}$ and high $S_{\mathrm{BET}}$ values were synthesized using lower $\mathrm{OCT} / \mathrm{H}_{2} \mathrm{O}$ ratios. Taking into account these results, we can suggest that under these synthesis conditions, CTAB participates mainly as template. The micelles are too small, so the hydrolytic condensation of 

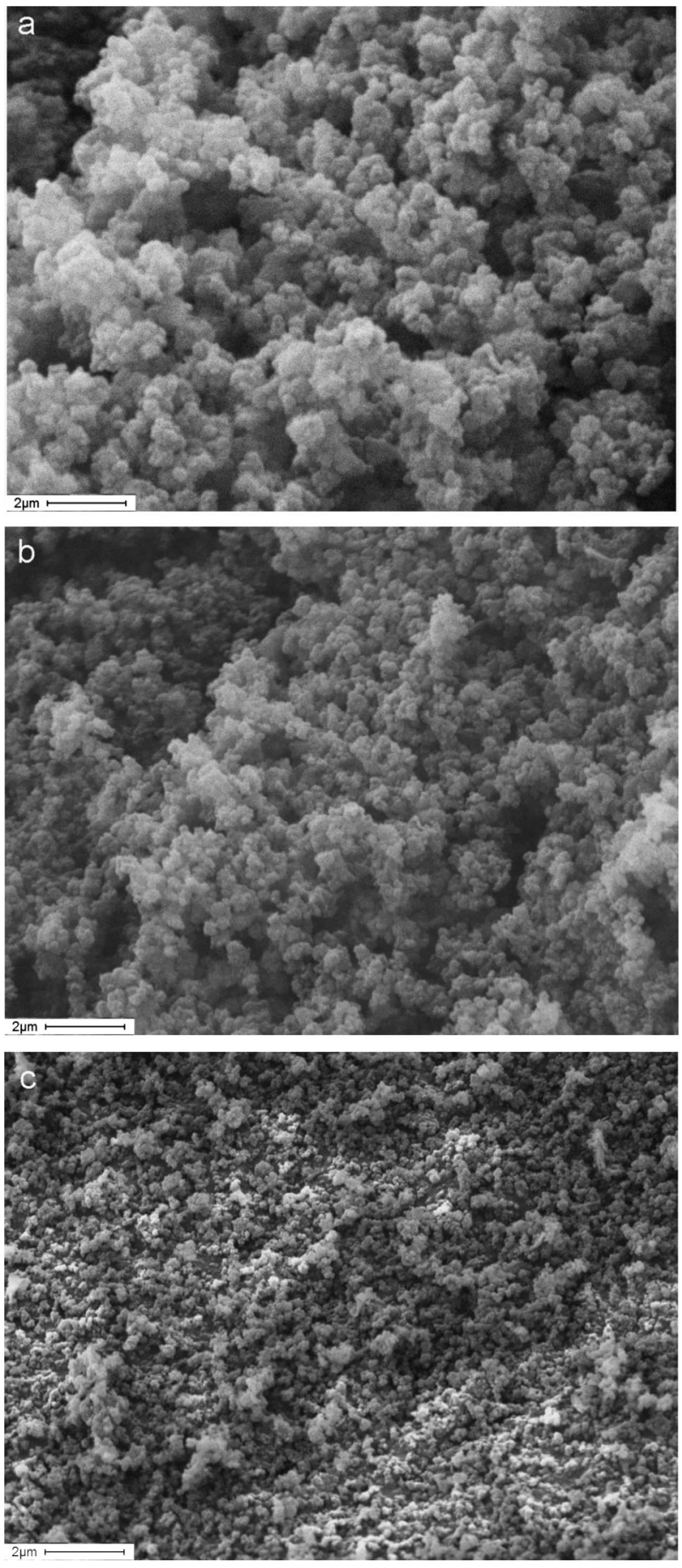

Fig. 2 SEM micrographs of a MSN1, b MSN2, and c MSN3 samples. Magnification: $5000 \times$; bar: $2 \mu \mathrm{m}$

tetraorthosilicate to form silica and the polymerization of styrene occur outside them. On the other hand, a higher OCT/ $\mathrm{H}_{2} \mathrm{O}$ ratio allows one to obtain mesoporous nanospheres with blackberry structure (size $\sim 32 \mathrm{~nm}$ ) displaying a bimodal PSD (mainly composed of large mesopores) with Dp in the range 12.9-13.5 nm and also high $S_{\text {BET }}$ values. In these cases, both silica and PS are produced within the large
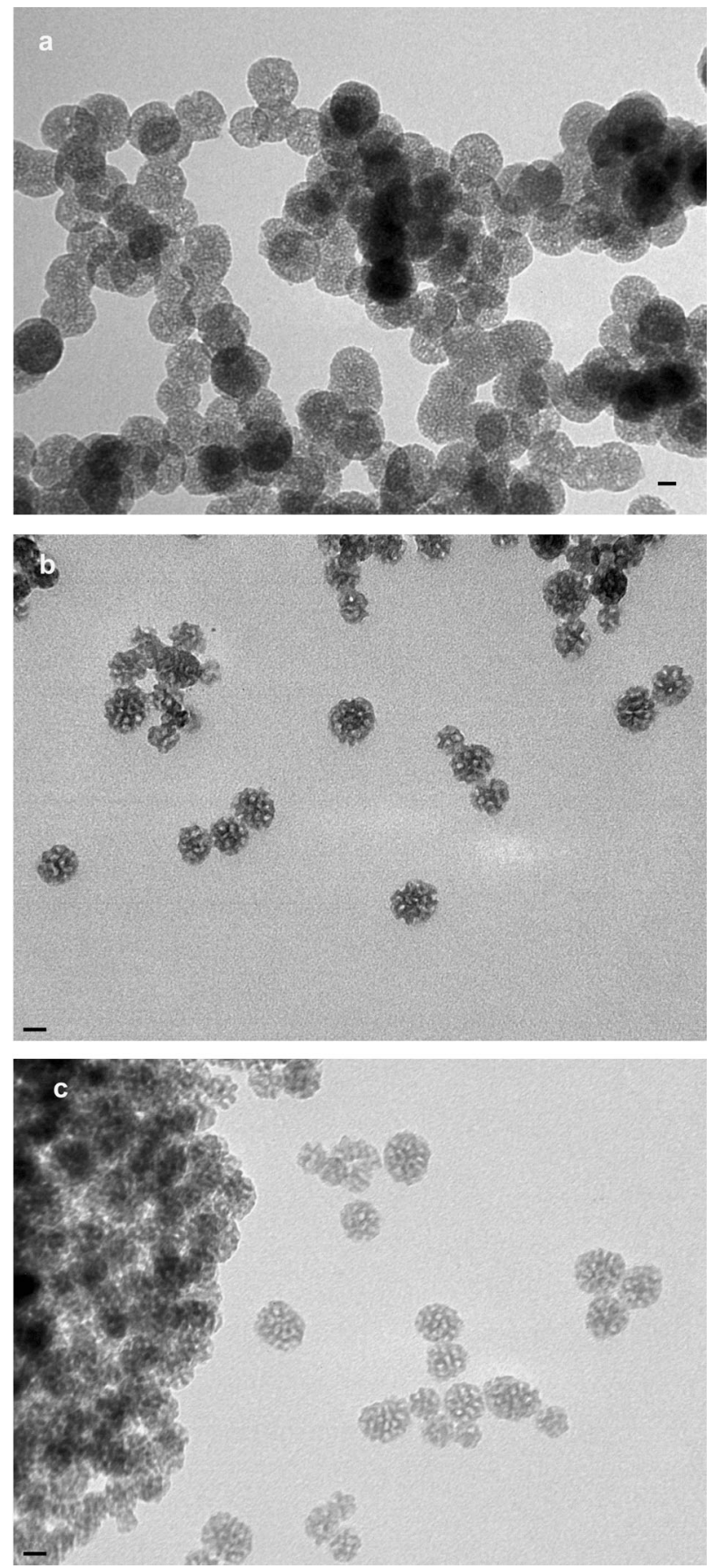

Fig. 3 TEM micrographs of a MSN1, b MSN3, and c MSN6 samples (bar: $20 \mathrm{~nm}$ )

micelles generated by the surfactant, housing the organic solvent inside them. The mesopores are mainly generated by PS, and a silica/PS composite structure was obtained.

The modification in styrene concentration led to a change not only in the size of the nanospheres but also in the mean pore diameter. However, its influence is not very strong, because an increase of the $\mathrm{EST} / \mathrm{H}_{2} \mathrm{O}$ ratio from 0.39 to 10 only reduces Dp by less than $15 \%$. 


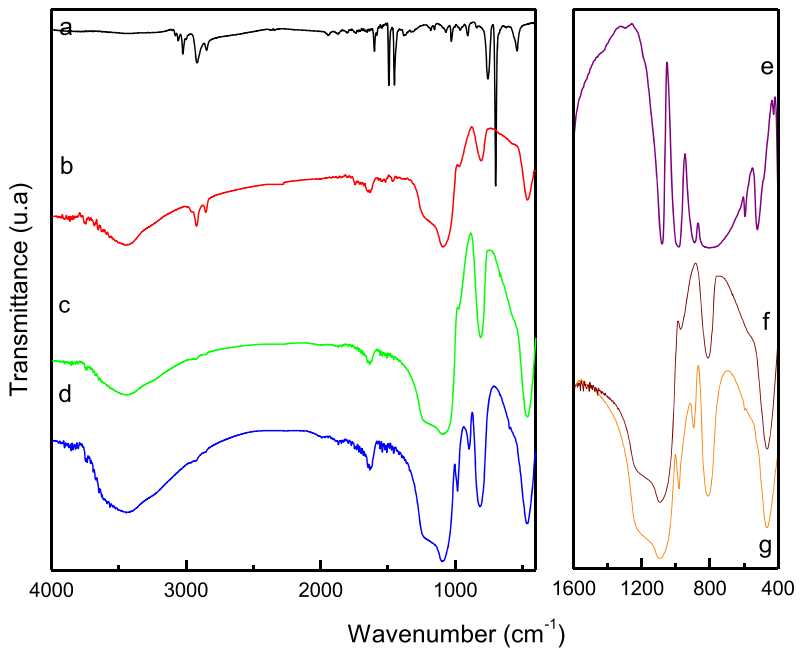

Fig. 4 FT-IR spectra of a polystyrene, b MSN1 without calcination, $\mathbf{c}$ MSN1 thermally treated at $500{ }^{\circ} \mathrm{C}$, d MSN1TPA, e TPA, f MSN3 thermally treated at $500^{\circ} \mathrm{C}$, and $\mathbf{g} \mathrm{MSN}_{3} \mathrm{TPA}_{\mathrm{C}}$

Then, the organic compounds were removed by the thermal treatment, which was corroborated by the FT-IR spectra of the materials before and after the heat treatment (Fig. 4), thereby obtaining the mesoporous structure.

The comparison of the spectra of the MSN1 before and after the thermal treatment (Fig. 4b, c) showed that the characteristic bands of PS, placed at 3080-2850, 1600, 1500, 750, 700, and $540 \mathrm{~cm}^{-1}$ (Fig. 4a), disappeared, thus indicating complete styrene removal. In these spectra, the characteristic bands of silica were observed at $3700-3200$ (stretching of $\mathrm{OH}$ groups), 1650 (angular vibration of $\mathrm{H}_{2} \mathrm{O}$ ), 1200-1100 (asymmetric stretching of the siloxane group), 965 (stretching of the $\mathrm{Si}-\mathrm{OH}$ group), 800 (stretching of the $\mathrm{Si}-\mathrm{O}-\mathrm{Si}$ group), and $480 \mathrm{~cm}^{-1}$ (bending of the $\mathrm{O}-\mathrm{Si}-\mathrm{O}$ group). On the other hand, the spectrum of the MSN1 sample impregnated with TPA (Fig. 4d) showed that the characteristic bands of TPA (Fig. 4e) were partially overlapped with the silica bands. However, the bands placed at 982 and $896 \mathrm{~cm}^{-1}$, attributed to the stretching of the $\mathrm{W}-\mathrm{O}_{\mathrm{d}}$ and $\mathrm{W}-\mathrm{O}-\mathrm{W}$ bonds, respectively, were clearly observed, thus indicating the presence of the tungstophosphate anion with unchanged Keggin structure [30]. The MSN3 (Fig. 4f, g) and the other materials showed similar characteristics to those described for MSN1 sample.

The ${ }^{31} \mathrm{P}$ MAS NMR spectrum of bulk hydrated TPA (Fig. 5a) $\left(\mathrm{H}_{3} \mathrm{PW}_{12} \mathrm{O}_{40} \cdot 6 \mathrm{H}_{2} \mathrm{O}\right)$ exhibits only one peak at $-15.3 \mathrm{ppm}$ with a small line width $(0.04 \mathrm{ppm})$. This peak was assigned to the deprotonated $\left[\mathrm{PW}_{12} \mathrm{O}_{40}\right]^{3-}$ Keggin anion [21] that interacts with $\mathrm{H}^{+}\left(\mathrm{H}_{2} \mathrm{O}\right)_{2}$ species [31, 32]. The ${ }^{31} \mathrm{P}$ MAS-NMR spectra of dried MSN1TPA $_{\mathrm{D}}$, MSN3TPA $_{D}$, and MSN6TPA $A_{D}$ samples (Fig. 5b-d, respectively) display two wide peaks with maximum at -15.2 and $-15.0 \mathrm{ppm}$, which were attributed to

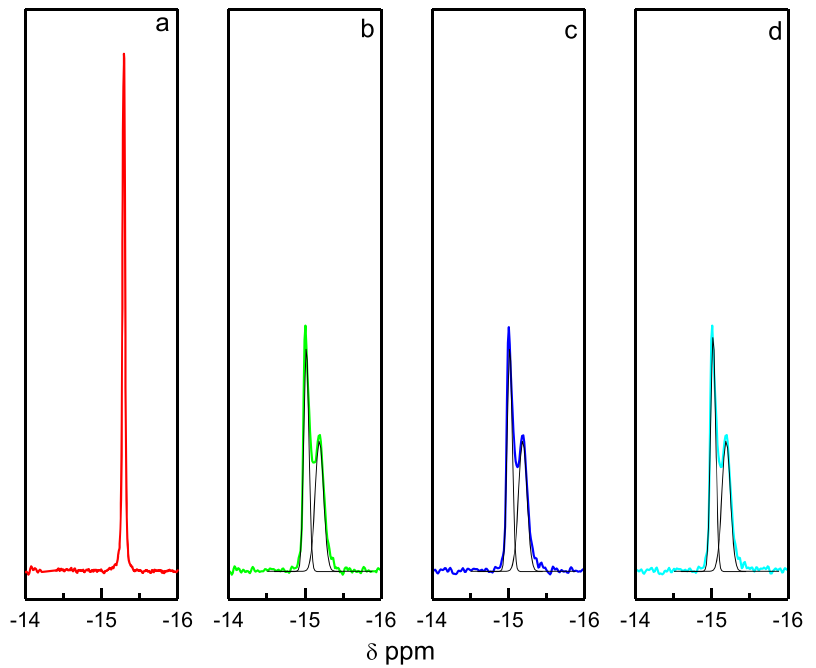

Fig. $5{ }^{31} \mathrm{P}$ MAS NMR spectra of $\mathrm{H}_{3} \mathrm{PW}_{12} \mathrm{O}_{40} \cdot 6 \mathrm{H}_{2} \mathrm{O}$ a, MSN1TPA $\mathbf{C}$, MSN3TPA ${ }_{C} \mathbf{c}$, and MSN6TPA ${ }_{C}$ d samples. The fitting results of $\left[\mathrm{PW}_{12} \mathrm{O}_{40}\right]^{3-}$ and $\left[\mathrm{H}_{3-X} \mathrm{PW}_{12} \mathrm{O}_{40}\right]^{(3-X)-}$ peaks are also shown

$\left[\mathrm{PW}_{12} \mathrm{O}_{40}\right]^{3-}$ interacting with $\mathrm{H}^{+}\left(\mathrm{H}_{2} \mathrm{O}\right)_{2}$ species (line width $0.08 \mathrm{ppm}$ ) and $\left[\mathrm{H}_{3-X} \mathrm{PW}_{12} \mathrm{O}_{40}\right]^{(3-X)-}$ (line width $0.12 \mathrm{ppm}$ ), respectively $[33,34]$. The downfield shift and the increase of the line width observed, compared to the TPA, can be ascribed to the interaction among the anions and the $\equiv \mathrm{Si}-\mathrm{OH}_{2}{ }^{+}$groups present in MSNX samples. The interaction can be assumed to be of the electrostatic type due to the transfer of protons to silanol groups, similarly to what has been proposed for the interaction with zirconia and titania $[1,34]$. The leaching experiments revealed that TPA is mostly strongly attached to the MSNX surface (less than $4 \%$ of the TPA total content was eliminated, suggesting that the interaction is very strong). On the other hand, the ${ }^{31} \mathrm{P}$ MAS-NMR spectra of the same samples after the calcination at $200^{\circ} \mathrm{C}$ (see Supplementary material) revealed the presence of two peaks with maximum at practically the same chemical shift ( -15.2 and $-14.9 \mathrm{ppm})$. However, the intensity of the peak assigned to the deprotonated $\left[\mathrm{PW}_{12} \mathrm{O}_{40}\right]^{3-}$ anion decreased in parallel with the rise of the signal ascribed to $\left[\mathrm{H}_{3-X} \mathrm{PW}_{12} \mathrm{O}_{40}\right]^{(3-X)-}$ anions. For example, the ratio between the intensity of the peaks assigned to $\left[\mathrm{PW}_{12} \mathrm{O}_{40}\right]^{3-}$ and $\left[\mathrm{H}_{3-X} \mathrm{PW}_{12} \mathrm{O}_{40}\right]^{(3-X)-}$ decreased from 1.70 to 1.15 when the MSN1TPA $A_{D}$ sample was treated at $200{ }^{\circ} \mathrm{C}$. The calcination of $\mathrm{H}_{3} \mathrm{PW}_{12} \mathrm{O}_{40} \cdot 6 \mathrm{H}_{2} \mathrm{O}$ at temperatures higher than $160{ }^{\circ} \mathrm{C}$ eliminates water molecules from $\mathrm{H}^{+}\left(\mathrm{H}_{2} \mathrm{O}\right)_{2}$ species and leads to the formation $\left[\mathrm{H}_{3} \mathrm{PW}_{12} \mathrm{O}_{40}\right]$ [35]. As a result of the thermal treatment at $200^{\circ} \mathrm{C}$, the amount of $\left[\mathrm{H}_{3-X} \mathrm{PW}_{12} \mathrm{O}_{40}\right]^{(3-\mathrm{X})-}$ anion interacting with $\equiv \mathrm{Si}-\mathrm{OH}_{2}{ }^{+}$groups increases according to the following transformation:

$$
\begin{gathered}
{\left[\mathrm{PW}_{12} \mathrm{O}_{40}\right]^{3-}+n \mathrm{H}^{+}\left(\mathrm{H}_{2} \mathrm{O}\right)_{2}+\equiv \mathrm{SiOH} \rightarrow} \\
\left(\equiv \mathrm{SiOH}_{2}\right)^{+}\left[\mathrm{H}_{3-\mathrm{X}} \mathrm{PW}_{12} \mathrm{O}_{40}\right]^{(3-X)-}+2 \mathrm{nH}_{2} \mathrm{O}
\end{gathered}
$$




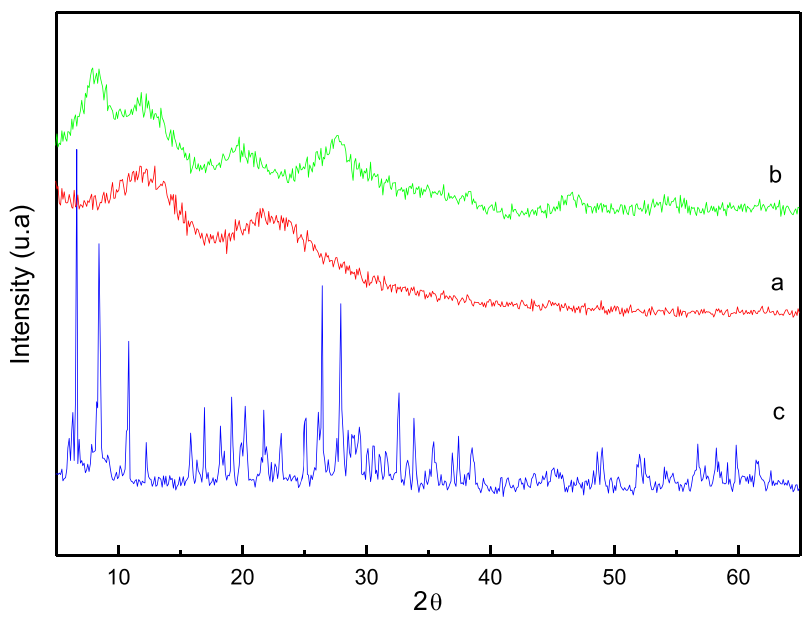

Fig. 6 DRX patterns of a MSN1 b MSN1TPA $_{C}$, and $\mathrm{H}_{3} \mathrm{PW}_{12} \mathrm{O}_{40} \cdot 23 \mathrm{H}_{2} \mathrm{O}$ c samples

So, the intensity of the ${ }^{31} \mathrm{P}$ MAS NMR band increases due to the formation of $\left(\equiv \mathrm{SiOH}_{2}^{+}\right)\left[\mathrm{H}_{3-\mathrm{X}} \mathrm{PW}_{12} \mathrm{O}_{40}\right]^{(3-\mathrm{X})-}$ species through electrostatic interaction increases.

For the material prepared by impregnation of the MSNX solids, it was observed that TPA addition did not lead to a significant change in the morphology and textural characteristics of the material used as support. However, although the mesoporous features of the MSNXTPA materials resembled those of the MSNX samples, their specific surface area decreased by about $25-30 \%$. The drop of the $S_{\mathrm{BET}}$ values can be explained considering that the specific surface area of bulk TPA is very low $\left(3-5 \mathrm{~m}^{2} / \mathrm{g}\right)$, and that MSNXTPA materials are composed of $30 \%$ of TPA.

The study of the solids by XRD displayed quite similar patterns for all the prepared samples showing, for example, those corresponding to MSN1 and $\mathrm{MSN} \mathrm{TPA}_{\mathrm{C}}$ materials (Fig. 6). The MSN1 sample presented two wide bands centered at around 12 and $22^{\circ} 2 \theta$ (Fig. 6a), characteristic of silica materials with amorphous structure, without peaks that can be assigned to the presence of crystalline phases. On the other hand, the patterns of the sample impregnated with TPA $\left(\right.$ MSN1TPA $\left._{C}\right)$ revealed the presence of three wide signals at around 8,19 , and $27^{\circ} 2 \theta$ (Fig. 6b), overlapped with those of the amorphous silica, without showing diffraction lines attributable to TPA crystalline phases such as $\mathrm{H}_{3} \mathrm{PW}_{12} \mathrm{O}_{40} \cdot 23 \mathrm{H}_{2} \mathrm{O}$ (Fig. 6c) or $\mathrm{H}_{3} \mathrm{PW}_{12} \mathrm{O}_{40} \cdot 6 \mathrm{H}_{2} \mathrm{O}$. These facts indicate that TPA is highly dispersed on the silica surface as a noncrystalline phase. This result can be due to the easy access of TPA species (whose mean size is $\sim 1.2 \mathrm{~nm}$ ) to the MSNX mesopores (Dp higher than $5.9 \mathrm{~nm}$ ) and the high surface area of these materials. Considering the cross-sectional area of the TPA anion to be equal to 1.13 $\mathrm{nm}^{2}$, we calculated that the percentage of total surface area of MSN samples "cover" by TPA ranged from 18 to $35 \%$ (for MSN6 and MSN3, respectively).

The EDX measurements revealed that the ratio between $\mathrm{Si} \mathrm{K} \alpha$ (at $1.74 \mathrm{KeV}$ ) and $\mathrm{W} \mathrm{L} \alpha(8.39 \mathrm{KeV})$ signal area (ASık/AWL) in MSNXTPA $_{C}$ samples was practically constant (in the range 8.70-8.90). EDX mapping images of $\mathrm{Si}, \mathrm{W}$, and $\mathrm{P}$ elements (Fig. 7) show that they are approximately homogeneously distributed in the MSNXTPA samples.

The low angle XRD patterns of MSNX samples did not show any peak $2 \theta$ values lower than $5^{\circ}$ such as those present in MCM-41 silica, synthesized using CTAB as template, indicating the formation of well-ordered mesoporous materials with hexagonal regularity $[36,37]$. The SAXS profiles (Fig. 8) were analyzed using the Guinier approximation [38]. For MSN3, MSN4, and MSN5 samples, the radius of gyration, obtained from the initial slope of the Guinier plot, was found to be $22 \pm 1 \mathrm{~nm}$, corresponding to a weight average particle radius of $\sim 31 \mathrm{~nm}$. In the case of MSN6 sample, the weight average particle radius obtained was lower $(27 \mathrm{~nm})$. The maximum at around $Q=1.28$ $\mathrm{nm}^{-1}$ was assigned to the presence of small particles $(\sim 4.7$ $\mathrm{nm})$ that compose the bigger ones. In the case of MSN1 and MSN2 samples, this maximum was not present. These values are in good agreement with those estimated from TEM.

On the other hand, no significant morphological changes were detected for the samples impregnated with TPA (both dried and calcined) by SAXS and TEM.

By using potentiometric titration with $n$-butylamine, it is possible to estimate the strength and the number of acid sites. The initial electrode potential (Ei) indicates the maximum strength, considering that values of $\mathrm{Ei}>100 \mathrm{mV}$ correspond to very strong sites, values of $\mathrm{Ei}$ in the range $100-0 \mathrm{mV}$ to strong sites, and values lower than $0 \mathrm{mV}$ to weak and very weak sites [39].

The area under the curve provides an estimation of the number of acid sites. For example, the titration curves of the MSN1, MSN3, and MSN6 samples (Fig. 9) reveal the presence of a low number of acid sites $(27,28$, and $24 \mathrm{meg}$ n-butylamine/g, respectively) with Ei values in the range 90-100. It was observed that the MSN materials impregnated with TPA dried at $70{ }^{\circ} \mathrm{C}\left(\mathrm{MSN}_{1 T P A} \mathrm{D}_{\mathrm{D}}, \mathrm{MSN}^{2} \mathrm{TPA} \mathrm{D}_{\mathrm{D}}\right.$, and MSN6TPA $A_{D}$ samples) present maximum acid strength values higher than that of the unmodified ones $(460,464$, and $461 \mathrm{mV}$, respectively). The number of acid sites of MSN1TPA $_{D}$, MSN3TPA $_{D}$, and MSN6TPA increases significantly $(138,142$, and $140 \mathrm{meg} n$-butylamine/g, respectively) as a result of TPA modification. However, the number of acid sites and their acid strength are practically the same for all the samples. On the other hand, both of them decrease as a result of the thermal 

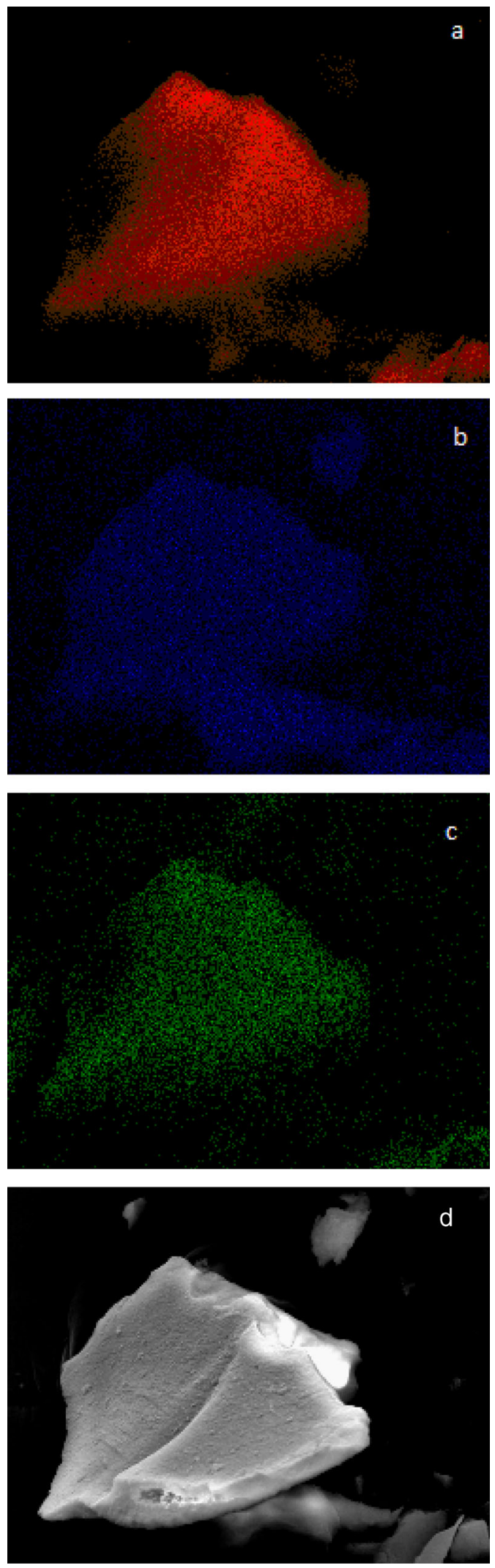

Fig. 7 Elemental mapping images of the MSN3TPA ${ }_{C}$ sample: a $\mathrm{Si}$ mapping, b W mapping, c P mapping, and d SEM image (Magnification 500x)
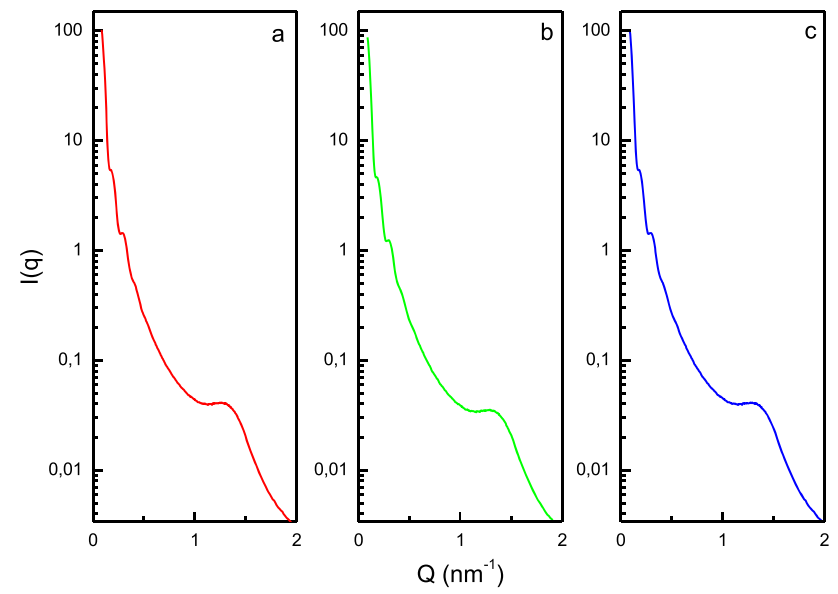

Fig. 8 Small angle X-ray scattering of MSN3 a, MSN4 b, and MSN5 c samples
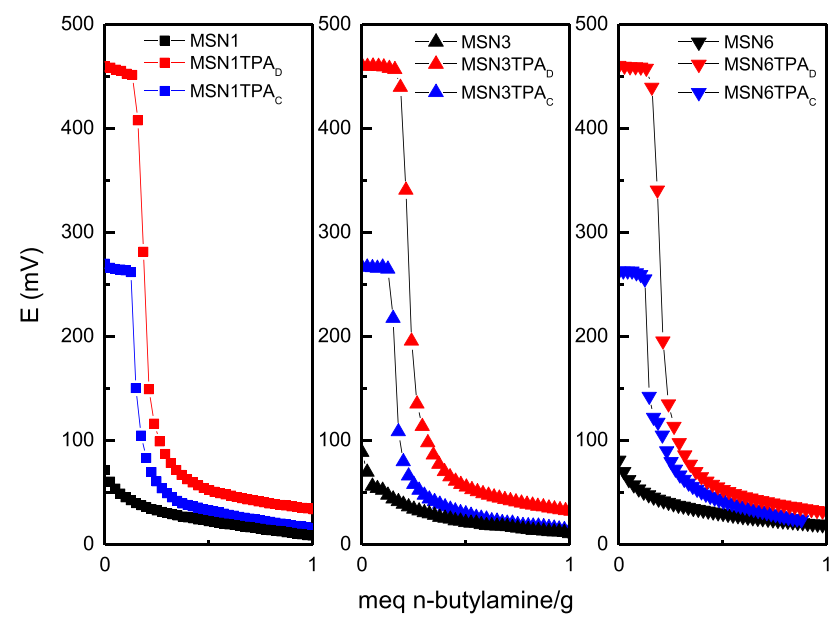

Fig. 9 Potentiometric titration curves of some MSNX, MSNXTPA and MSNXTPA $_{C}$ representative samples

treatment at $200^{\circ} \mathrm{C}$, but remain higher than those obtained for the unmodified silica.

As was mentioned before, the loss of water molecules and the increment of $\left(\equiv \mathrm{SiOH}_{2}^{+}\right)\left[\mathrm{H}_{3-\mathrm{X}} \mathrm{PW}_{12} \mathrm{O}_{40}\right]^{(3-\mathrm{X})-}$ species during the thermal treatment can be one of the reasons for the decrease in the acid properties of the calcined samples. The other could be the removal of a water molecule from $\left(\equiv \mathrm{SiOH}_{2}{ }^{+}\right)\left[\mathrm{H}_{3-\mathrm{X}} \mathrm{PW}_{12} \mathrm{O}_{40}\right]^{(3-\mathrm{X})-}$ species and the formation of $\mathrm{Si}-\mathrm{O}-\mathrm{W}$ bonds.

In sum, mesoporous silica nanoparticle materials appropriate to be used as support of catalytic compounds were prepared. The impregnation of these solids with TPA allowed maintaining the Keggin structure of the acid, so leading to high acidic materials. We consider these materials suitable to be used as catalyst in the synthesis of quinoxaline derivatives as they have better textural and acid properties than those previously tested [4]. Preliminary results obtained from the synthesis of 2,3-diphenylpyrido 
[2,3-b]pyrazine using MSN1TPA ${ }_{C}$, and MSN6TPA Mam- $_{C}$ ples as catalysts (see the experimental conditions employed in Supplementary material) confirmed that assumption. The times necessary to complete the reaction between 1,2-diaminobenzene and 2,3-diamine pyridine using MSN1TPA $_{\mathrm{C}}$ and MSN6TPA $\mathrm{C}_{\mathrm{C}}$ as catalyst (105 and $95 \mathrm{~min}$, respectively) were shorter than the one previously reported $(120 \mathrm{~min})$ for the same reaction catalyzed by TPA included in zirconia [4]. Taking into account that MSN1TPA ${ }_{C}$ and MSN6TPA samples present similar acidic properties, the better catalytic performance of the latter can be due to its higher specific surface area and mean pore diameter values.

\section{Conclusions}

Mesoporous silica nanospheres with blackberry structure were prepared in aqueous/organic phase by the template method. The OCT/ $\mathrm{H}_{2} \mathrm{O}$ ratio influences the PSD, the morphology and size of the nanospheres obtained. All the MSN materials display a bimodal mesopore distribution. The relative contribution of small (in the range 3-6 nm) and large (in the range $28-34 \mathrm{~nm}$ ) mesopores to the specific surface area and total pore volume of the solids can be easily tuned by varying octane and styrene concentration. In the materials impregnated with TPA, the FT-IR and ${ }^{31} \mathrm{P}$ MAS NMR characterization revealed the presence of the undegraded $\left[\mathrm{PW}_{12} \mathrm{O}_{40}\right]^{3-}$ and $\left[\mathrm{H}_{3-X} \mathrm{PW}_{12} \mathrm{O}_{40}\right]^{(3-X)-}$ species interacting electrostatically with the $\equiv \mathrm{Si}-\mathrm{OH}_{2}{ }^{+}$groups present in the MSN support. On the other hand, potentiometric titration showed the presence of very strong acid sites in the MSNTPA solids.

Materials based on TPA impregnated on MSN showed excellent textural properties and very strong acidic characteristics. All the results indicate that the prepared materials will be suitable for their use as catalysts in acid reactions, especially to obtain heterocyclic organic compounds such as quinoxaline derivatives.

Acknowledgements The authors acknowledge L. Osiglio, G. Valle, $\mathrm{P}$. Fetsis and M. Theiller for their experimental contribution and CONICET (PIP 628) and UNLP (X638 and X732) for the financial support.

\section{Compliance with ethical standards}

Conflict of interest The authors declare that they have no competing interests.

\section{References}

1. Rivera TS, Sosa A, Romanelli GP, Blanco MN, Pizzio LR (2012) Tungstophosphoric acid/zirconia composites prepared by the sol-gel method: an efficient and recyclable green catalyst for the one-pot synthesis of 14-aryl-14H-dibenzo[a,j] xanthenes. Appl Catal A 443-444:207-213

2. Rengifo-Herrera JA, Frenzel RA, Blanco MN, Pizzio LR (2014) Visible-light-absorbing mesoporous $\mathrm{TiO}_{2}$ modified with tungstosilicicacid as photocatalyst in the photodegradation of 4chlorophenol. J Photochem Photobiol A 289:22-30

3. Nandiyanto ABD, Hagura N, Iskandar F, Okuyama K (2010) Design of a highly ordered and uniform porous structure with multisized pores in film and particle forms using a template-driven self-assembly technique. Acta Mater 58:282-289

4. Sosa A, Rivera TS, Blanco MN, Pizzio LR, Romanelli GP (2013) Tungstophosphoric acid supported on zirconia: a recyclable catalyst for the green synthesis on quinoxaline derivatives under solvent-free conditions. Phosphorus Sulfur Silicon Relat Elem 188:1071-1079

5. Iskandar F, Lenggoro IW, Kim TO, Nakao N, Shimada M, Okuyama K (2001) Fabrication and characterization of $\mathrm{SiO}_{2}$ particles generated by spray method for standards aerosol. J Chem Eng Jpn 34:1285-1292

6. Ray S, Bhaumik A, Pramanik M, Butcher RJ, Yildirim SO, Mukhopadhyay C (2014) Binary conjugate Brønsted-Lewis acid supported on mesoporous silica nanoparticles for the domino addition/elimination/addition and addition/elimination/addition/ cyclization cascade. Catal Commun 43:173-178

7. Tu J, Wang T, Shi W, Wu G, Tian X, Wang Y, Ge D, Ren L (2012) Multifunctional ZnPc-loaded mesoporous silica nanoparticles for enhancement of photodynamic therapy efficacy by endolysosomal escape. Biomaterials 33:7903-7914

8. Mehraban Z, Farzaneh F (2006) MCM-41 as selective separator of chlorophyll-a from b-carotene and chlorophyll- $a$ mixture. Microporous Mesoporous Mater 88:84-90

9. Hosseini M, Ganjali MR, Aboufazeli F, Faridbod F, Goldooz H, Badiei A, Norouzi P (2013) A selective fluorescent bulk sensor for lutetium based on hexagonal mesoporous structures. Sens Actuators B 184:93-99

10. Kamyshny A, Magdassi S (2000) Fluorescence immunoassay based on fluorescer microparticles. Colloids Surf B 18:13-17

11. Yu J, Shi JL, Chen HR, Yan JN, Yan DS (2001) Effect of inorganic salt addition during synthesis on pore structure and hydrothermal stability of mesoporous silica. Microporous Mesoporous Mater 46:153-162

12. Nandiyanto ABD, Kim SG, Iskandar F, Okuyama K (2009) Synthesis of spherical mesoporous silica nanoparticles with nanometer-size controllable pores and outer diameters. Microporous Mesoporous Mater 120:447-453

13. Rossi LM, Shi L, Quina FH, Rosenzweig Z (2005) Stöber synthesis of monodispersed luminescent silica nanoparticles for bioanalytical assays. Langmuir 21:4277-4280

14. Burns A, Vider J, Ow H, Herz E, Penate-Medina O, Baumgart M, Larson SM, Wiesner U, Bradbury M (2009) Fluorescent silica nanoparticles with efficient urinary excretion for nanomedicine. Nano Lett. 9:442-448

15. Heikkila T, Salonen J, Tuura J, Hamdy MS, Mul G, Kumar N, Salmi T, Yu D, Murzin D, Laitinen L, Kaukonen AM, Hirvonen J, Lehto VP (2007) Mesoporous silica material TUD-1 as a drug delivery system. Int J Pharm 331:133-138

16. Keggin JF (1934) The structure and formula of 12- phosphotungstic acid. Proc R Soc London Ser A 144:75-100

17. Rao KM, Gobetto R, Iannibello A, Zecchina A (1989) Solid state NMR and IR studies of phosphomolybdenum and phosphotungsten heteropoly acids supported on $\mathrm{SiO}_{2}, \alpha-\mathrm{Al}_{2} \mathrm{O}_{3}$, and $\mathrm{SiO}_{2}-\mathrm{Al}_{2} \mathrm{O}_{3}$. J Catal 119:512-516

18. Kapustin GI, Brueva TR, Klyachkov AL, Timofeeva MN, Kulikov SM, Kozhevnikov IV (1991) Study of the acidity of heteropolyacids. Kinet Catal 31:1017-1020 
19. Moffat JB, Kasztelan S (1988) The oxidation of methane on heteropolyoxometalates II. Nature and stability of the supported species. J Catal 109:206-211

20. Kasztelan S, Payen E, Moffat JB (1988) The formation of molybdosilicic acid on $\mathrm{Mo} / \mathrm{SiO} 2$ catalysts and its relevance to methane oxidation. J Catal 112:320-324

21. Lefebvre $\mathrm{F}(1992){ }^{31} \mathrm{P}$ MAS NMR study of $\mathrm{H}_{3} \mathrm{PW}_{12} \mathrm{O}_{40}$ supported on silica: formation of $\left(\equiv \mathrm{SiOH}_{2}{ }^{+}\right)\left(\mathrm{H}_{2} \mathrm{PW}_{12} \mathrm{O}_{40}{ }^{-}\right)$. J Chem Soc Chem Commun (10): 756-757

22. Legagneux N, Basset JM, Thomas A, Lefebvre F, Goguet A, Sa J, Hardacre Ch (2009) Characterization of silica-supported dodecatungstic heteropolyacids as a function of their dehydroxylation temperature. Dalton Trans (12): 2235-2240

23. Pizzio LR, Cáceres CV, Blanco MN (1998) Acid catalysts prepared by impregnation of tungstophosphoric acid solutions on different supports. Appl Catal A 167:283-294

24. Okuhara T, Mizuno N, Misono M (1996) Catalytic chemistry of heteropoly compounds. Adv Catal 41:113-252

25. Jeannin YP (1998) The nomenclature of polyoxometalates: how to connect a name and a structure. Chem Rev 98:51-76

26. Kozhevnikov IV (1998) Catalysis by heteropoly acids and multicomponent polyoxometalates in liquid-phase reactions. Chem Rev 98:171-198

27. Misono M (2001) Unique acid catalysis of heteropoly compounds (heteropolyoxometalates) in the solid state. Chem Commun (13): $1141-1152$.

28. Anastas PT, Warner JC (1998) Green chemistry: theory and practice. Oxford University Press, Oxford

29. Ajaikumar S, Pandurangan A (2007) Esterification of alkyl acids with alkanols over MCM-41 molecular sieves: influence of hydrophobic surface on condensation reaction. J Mol Catal A 266:1-10

30. Rocchiccioli-Deltcheff C, Thouvenot R, Franck R (1976) Spectres i.r. et Raman d'hétéropolyanions $\alpha-\mathrm{XM}_{12} \mathrm{O}_{40}{ }^{\mathrm{n}-}$ de structure de type $\operatorname{Keggin}\left(\mathrm{X}=\mathrm{B}^{\mathrm{III}}, \mathrm{Si}^{\mathrm{IV}}, \mathrm{Ge}^{\mathrm{IV}}, \mathrm{P}^{\mathrm{V}}, \mathrm{As}^{\mathrm{V}}\right.$ et $\mathrm{M}=\mathrm{W}^{\mathrm{VI}}$ et $\left.\mathrm{Mo}^{\mathrm{VI}}\right)$. Spectrochim Acta Part A 32:587-597

31. Essayem N, Tong YY, Jobic H, Vedrine JC (2000) Characterization of protonic sites in $\mathrm{H}_{3} \mathrm{PW}_{12} \mathrm{O}_{40}$ and $\mathrm{Cs}_{1.9} \mathrm{H}_{1.1} \mathrm{PW}_{12} \mathrm{O}_{40}$ : a solid-state ${ }^{1} \mathrm{H},{ }^{2} \mathrm{H},{ }^{31} \mathrm{P}$ MAS-NMR and inelastic neutron scattering study on samples prepared under standard reaction conditions. Appl Catal A 194-195:109-122

32. Okuhara T, Nishimura T, Watanabe H, Na K, Misono M (1994) 4.8 novel catalysis of cesium salt of heteropoly acid and its characterization by solid-state NMR. Stud Surf Sci Catal 90:419-428

33. Massart R, Contant R, Fruchart J, Ciabrini J, Fournier M (1977) Phosphorus-31 NMR studies on molybdic and tungstic heteropolyanions. Correlation between structure and chemical shift. Inorg Chem 16:2916-2921

34. Rengifo-Herrera JA, Blanco MN, Wist J, Florian P, Pizzio LR (2016) $\mathrm{TiO}_{2}$ modified with polyoxotungstates should induce visible-light absorption and high photocatalytic activity through the formation of surface complexes. App Catal A 189:99-109

35. Rengifo-Herrera JA, Blanco MN, Fidalgo de Cortalezzi MM, Pizzio LR (2016) Visible-light-absorbing Evonik P-25 nanoparticles modified with tungstophosphoric acid and their photocatalytic activity on different wavelengths. Mater Res Bull 83:360-368

36. Chen CY, Li HX, Davis ME (1993) Studies on mesoporous materials: I. Synthesis and characterization of MCM-41. Microporous Mater 2:17-26

37. Zhao XS, Lu GQ, Millar GJ (1996) Synthesis and characterization of highly ordered MCM-41 in an alkali-free system and its catalytic activity. Catal Lett 38:33-37

38. Li T, Senesi AJ, Lee B (2016) Small angle X-ray scattering for nanoparticle research. Chem Rev 116:11128-11180

39. Pizzio LR, Cáceres CV, Blanco MN (1999) Equilibrium adsorption of 11-tungstophosphate anion on different supports. Appl Surf Sci 151:91-101 